\title{
SEMIGROUPS AND STABILITY OF NONAUTONOMOUS DIFFERENTIAL EQUATIONS IN BANACH SPACES
}

\author{
NGUYEN VAN MINH
}

\begin{abstract}
This paper is concerned with nonautonomous differential equations in Banach spaces. Using the theory of semigroups of linear and nonlinear operators one investigates the semigroups of weighted translation operators associated with the underlying equations. Necessary and sufficient conditions for different types of stability are given in terms of spectral properties of the translation operators and the differential operators associated with the equations.
\end{abstract}

\section{INTRODUCTION}

Let us consider the equation

$$
\frac{d x}{d t}=A(t) x,
$$

where $t \in J, J=[0,+\infty)$ or $(-\infty,+\infty), x \in \mathbb{X}, \mathbb{X}$ is a given complex Banach space, $A(\cdot)$ is an operator valued function taking $t$ into a bounded linear operator $A(t)$ acting on $\mathbb{X}$. It is known that in the autonomous case, i.e. $A(t)=A$ for all $t \in \mathbb{R}$, the theory of linear autonomous differential equations is based on the investigation of spectral properties of the operator $A$ (see e.g. [4]). In this case, the Dunford Theorem plays a key role which allows one to establish the relationship between the spectrum of the operator $A$ and that of the one-parameter group $\exp [t A], t \in \mathbb{R}$. A higher level of difficulty occurs when one is concerned with nonautonomous equations, i.e. $A(t)$ depends on $t$. Many of the classical results concerning the asymptotic behavior of solutions of equation (1) are characterized in terms of the existence of bounded solutions to this equation (see e.g. $[4,6]$ ). From the point of view of the operator theory the above characterization is in fact in terms of spectral properties of the differential operator $\mathscr{L}=-d / d t+A(t)$ acting on suitable function spaces. It turns out that the operator $\mathscr{L}$ constitutes the infinitesimal generator of the semigroup $\mathscr{T}=\left\{T^{h}, h \geq 0\right\}$ defined as follows

$$
\left(T^{h} v\right)(t)=X(t, t-h) v(t-h), \quad(t \in \mathbb{R}),
$$

acting on suitable subspace of the space of bounded and continuous functions.

In [1], [10] the authors considered individual operators of the above semigroup acting on the space of bounded functions. It was shown that one can

Received by the editors June 25, 1992 and, in revised form, September 10, 1993.

1991 Mathematics Subject Classification. Primary 34G05, 34D05; Secondary 47D05, 47H20.

Key words and phrases. Weighted translation operator, semigroup, differential operator, uniform stability, exponential dichotomy, stability of individual solutions. 
investigate the asymptotic behavior of solutions of equation (1) by studying spectral properties of the operator $T^{h}$ for some given positive $h$. In [10] it was announced that one could relate the spectral properties of the semigroup $\mathscr{T}$ to those of differential operator $\mathscr{L}$ in the context of the theory of linear semigroups. The main purpose of this paper is to investigate different types of stability by studying the relationship between the operators $\mathscr{L}$ and $\mathscr{T}$ by means of the theory of semigroups of linear and nonlinear operators. More concretely, one will consider the action of the semigroup $\mathscr{T}$ on different suitable function spaces and then prove the spectral mapping theorems, i.e. the following equality

$$
\sigma\left(T^{h}\right)=\exp [h \sigma(\mathscr{L})]
$$

where $\sigma(\cdot)$ denotes the spectrum of linear operator. Our investigation is also carried over nonlinear equations

$$
d x / d t=f(t, x),
$$

by means of the theory of nonlinear operators. Part of the paper's result was announced in [7-8].

We now give an outline of the contents of the paper. In $\S I I$ we will recall some notions and notations which will be used throughout the paper. Section III is devoted to the study of equations (1) and (4) on the whole line. Lemma 1 shows that the differential operator $\mathscr{L}=-d / d t+f(t, \cdot)$ is the infinitesimal generator of the nonlinear semigroup $\mathscr{T}$. This assertion will be used throughout the paper. The main result concerning the stability of nonlinear equations is Theorem 2 in which a sufficient condition for the existence and global exponential stability of a solution to the nonlinearly perturbed equation of (4) is stated in terms of the accretiveness of the operator $\mathscr{L}=-d / d t+f(t, \cdot)$. In this section we study equation (1) by establishing the relation between the spectrum of $\mathscr{L}=-d / d t+A(t)$ and that of the semigroup $\mathscr{T}$. We shall prove (3) for the case $\operatorname{dim} \mathbb{X}<\infty$ from which we deduce necessary and sufficient conditions for the exponential dichotomy of equation (1). In the case $\operatorname{dim} \mathbb{X}=\infty$ a sufficient condition for the asymptotic stability of individual solutions of equation (1) is given by applying a recent result due to Batty and $\mathrm{Vu}$ [2]. Necessary and sufficient conditions for the exponential and uniform stabilities are also obtained and stated in terms of spectral properties of the operators $\mathscr{L}$ and $T^{h}$. In $\S I V$ we shall investigate equation (1) defined on the half line $[0,+\infty)$. A detailed study of the spectra of the semigroups $\mathscr{T}$ and $\mathscr{K}=\left\{T^{-h}, h \geq 0\right\}$ acting on suitable function spaces is carried out. From this we deduce necessary and sufficient conditions for the upper and lower Bohl exponents of equation (1) to be negative and positive respectively. Similarly to the case where equation (1) is defined on the whole line, in this case we give necessary and sufficient conditions for different types of stability in terms of spectral properties of the operator $\mathscr{L}$ and the semigroups $\mathscr{T}$ and $\mathscr{K}$.

\section{NOTATIONS AND DEFINITIONS}

In the paper $\mathbb{X}$ always denotes a given complex Banach space with norm $\|\cdot\|$. We shall denote by $C_{u}(J, \mathbb{X})$ the Banach space of all bounded and uniformly continuous functions from $J$ to $\mathbb{X}$ with norm

$$
\|v\|=\sup _{t \in J}\|v(t)\|
$$


where $v$ is any element of $C_{u}(J, \mathbb{X})$. For a linear operator $A$ acting on a Banach space $\mathbb{Y}$ we shall denote by $\mathscr{D}(A)$ and $\mathscr{R}(A)$ the domain and range of $A$, respectively. If $A$ is bounded then $\|A\|$ will stand for its norm. $\sigma(A), \rho(A)$ and $r_{\sigma}(A)$ denote the spectrum, resolvent and spectral radius of $A$, respectively.

Throughout the paper the operator valued function $A(\cdot)$ is always assumed to be continuous on $J$. So from the Existence and Uniqueness Theorem it follows that the evolutionary operator $X(t, s)$ of equation (1) exists for all $t, s \in J$.

Definition $1[4,6]$. Equation (1) is said to have an exponential dichotomy on $J$ if there exist positive constants $K, \alpha$ and a continuous projection $P$ in $\mathbb{X}$ such that the following inequalities hold

$$
\begin{gathered}
\left\|X(t) P X^{-1}(s)\right\| \leq K \exp [-\alpha(t-s)], \text { for } t \geq s, \\
\left\|X(t)(I-P) X^{-1} X(s)\right\| \leq K \exp [-\alpha(s-t)], \text { for } s \geq t,
\end{gathered}
$$

where $X(t)$ denotes $X(t, 0)$.

Definition $2[4,6]$. Equation (1) is said to be uniformly stable if there exists a positive constant $N$ such that

$$
\|X(t, s) x\| \leq N
$$

for all $t \geq s, x \in \mathbb{X}$.

Definition 3. For an arbitrary Banach space $\mathbb{Y}$, a nonlinear operator $A$ from $\mathbb{Y}$ to $\mathbb{Y}$ is said to be accretive if the following inequality holds

$$
\|(I+\lambda A) x-(I+\lambda A) y\| \geq\|x-y\|
$$

for all $x, y \in \mathscr{D}(A), \lambda>0$.

Suppose that equation (4) satisfies all conditions of the Existence and Uniqueness Theorem and all its solutions are continuable on the whole line.

Definition 4. A solution $x_{0}(t)$ to equation (4) is said to be globally exponentially stable if there exist positive constants $N, \alpha$ such that the inequality

$$
\left\|x(t)-x_{0}(t)\right\| \leq N \exp [-\alpha(t-s)]\left\|x(s)-x_{0}(s)\right\|
$$

holds for all $t \geq s$ and any solution $x(t)$ of equation (4). For linear equations the exponential stability means the global exponential stability.

\section{EQUATIONS ON THE WHOLE LINE}

In this section we shall consider linear and nonlinear equations defined on the whole line. Using the spectral theory of linear semigroups we shall prove the spectral mapping theorem, and then give necessary and sufficient conditions for equation (1) to have an exponential dichotomy in the case $\operatorname{dim} \mathbb{X}<\infty$. In the general case we obtain necessary and sufficient conditions for the uniform and asymptotic uniform stability of solutions as well as a sufficient condition for the asymptotic stability of individual solutions. For nonlinear equations we shall apply a general theory on the generation of nonlinear semigroups due to Crandall-Liggett [3] to give a sufficient condition for the existence of a globally exponentially stable solution of nonlinearly perturbed equations. In order to organize the paper optimally we first deal with the nonlinear equation (4). 
Definition 5. A mapping $f$ from $\mathbb{R} \times \mathbb{X}$ to $\mathbb{X}$ is said to be admissible if it satisfies the following conditions:

(i) $f(t, x)$ is continuous with respect to $(t, x)$,

(ii) $f(t, x)$ satisfies the Lipschitz condition with respect to $x$ with coefficient independent of $t$, i.e. there is a positive constant $L$ such that

$$
\|f(t, x)-f(t, y)\| \leq L\|x-y\|
$$

for all $t \in \mathbb{R}$ and $x, y \in \mathbb{X}$,

(iii) There is a positive constant $N$ such that

$$
\|f(t, x)\| \leq N(1+\|x\|)
$$

for all $t \in \mathbb{R}$ and $x \in \mathbb{X}$.

Definition 6. We say that a mapping $f$ from $\mathbb{R} \times \mathbb{X}$ to $\mathbb{X}$ satisfies condition $H$ if it is admissible and satisfies the following condition: for every $v \in C_{u}(\mathbb{R}, \mathbb{X})$ the function taking $t$ into $f(t, v(t))$ belongs to $C_{u}(\mathbb{R}, \mathbb{X})$ as well.

If $f$ is admissible, then equation (4) satisfies all conditions of the Existence and Uniqueness Theorem [4]. Furthermore all its solutions are continuable on the whole line. Denoting by $X(t, s) x$ the solution to the Cauchy problem

$$
\left\{\begin{array}{l}
\frac{d x}{d t}=f(t, x) \\
x(s)=x
\end{array}\right.
$$

We shall use the formula (2) to define the group of weighted nonlinear translation operators $T^{h}, h \in \mathbb{R}$, associated with equation (4). In what follows in order to apply the theory of nonlinear semigroups we shall deal with the semigroups $\mathscr{T}=\left\{T^{h}, h \geq 0\right\}$.

Lemma 1. Assume that $f$ is admissible. Then every operator $T^{h}$ of the semigroup $\mathscr{T}$ associated with equation (4) acts on $C_{u}(\mathbb{R}, \mathbb{X})$ with $\mathscr{D}\left(T^{h}\right)=C_{u}(\mathbb{R}, \mathbb{X})$. In addition the semigroup $\mathscr{T}$ is strongly continuous, i.e. for every given $v \in$ $C_{u}(\mathbb{R}, \mathbb{X})$

$$
\lim _{h \rightarrow 0} T^{h} v=v .
$$

Proof. From the definition of $X(t, s)$ and the admissibility of $f$ (see (5) and (6)) we have

$$
\|X(t, s) x\| \leq\|x\|+\int_{s}^{t} N(1+\|X(\xi, s) x\|) d \xi
$$

for all $t \geq s, t, s \in \mathbb{R}, x \in \mathbb{X}$. Applying the Gronwall inequality we get the following estimate

$$
\|X(t, s) x\| \leq(\|x\|+N(t-s)) e^{N(t-s)},
$$

for all $t \geq s, x \in \mathbb{X}$. By definition we have

$$
\begin{aligned}
\left\|\left(T^{h} v\right)(t)\right\| & =\|X(t, t-h) v(t-h)\| \\
& \leq\|v(t-h)\|+\int_{t-h}^{t} N(1+\|X(\xi, t-h) v(t-h)\|) d \xi .
\end{aligned}
$$


Substituting the estimate (10) into (11) we obtain

$$
\begin{aligned}
\left\|\left(T^{h} v\right)(t)\right\| & \leq\|v\|+\int_{t-h}^{t} N\left[1+(\|v(t-h)\|+N(\xi-t+h)) e^{N(\xi-t+h)}\right] d \xi \\
& \leq\left\{\|v\|+N h+\|v\|\left[e^{N h}-1\right]+N h e^{N h}-\left[e^{N h}-1\right]\right\} .
\end{aligned}
$$

From(12) we deduce the boundedness of $T^{h} v$. We are going to prove the uniform continuity of $T^{h} v$. From the admissibility of $f$ we get

$$
\|X(t, s) x-X(t, s) y\| \leq\|x-y\|+\int_{s}^{t} L\|x(\xi, s) x-X(\xi, s) y\| d \xi
$$

for all $t \geq s, x, y \in \mathbb{X}$. Applying the Gronwall inequality we obtain the estimate

$$
\|X(t, s) x-X(t, s) y\| \leq e^{L(t-s)}\|x-y\|
$$

for all $t \geq s, x, y \in \mathbb{X}$. We have

$$
\begin{gathered}
\left\|\left(T^{h} v\right)(t)-\left(T^{h} v\right)(s)\right\|=\|X(t, t-h) v(t-h)-X(s, s-h) v(s-h)\| \\
\leq\|X(t, t-h) v(t-h)-X(t, t-h) v(s-h)\| \\
\quad+\|X(t, t-h) v(s-h)-X(s, s-h) v(s-h)\| .
\end{gathered}
$$

From (14) and the uniform continuity of $v$ it follows that

$$
\lim _{t-s \rightarrow 0}\|X(t, t-h) v(t-h)-X(t, t-h) v(s-h)\|=0 .
$$

On the other hand, without loss of generality, assuming that $t \geq s$ and $|t-s|$ is sufficiently small we have

(17)

$$
\begin{aligned}
\| X(t, & t-h) v(s-h)-X(s, s-h) v(s-h) \| \\
\leq & \int_{s}^{t} \| f(\xi, X(\xi, t-h) v(s-h) \| d \xi \\
& +\int_{t-h}^{s}\|f(\xi, X(\xi, t-h) v(s-h))-f(\xi, X(\xi, s-h) v(s-h))\| d \xi \\
& +\int_{s-h}^{t-h}\|f(\xi, X(\xi, s-h) v(s-h))\| d \xi .
\end{aligned}
$$

To show that the right-hand side of (17) tends to zero as $t-s$ tends to zero it suffices to show that

$$
\lim _{t-s \rightarrow 0} \sup _{t-h \leq \xi \leq s}\|X(\xi, t-h) v(s-h)-X(\xi, s-h) v(s-h)\|=0 .
$$

We have

$$
\begin{aligned}
& \sup _{t-h \leq \xi \leq s}\|X(\xi, t-h) v(s-h)-X(\xi, s-h) v(s-h)\| \\
& \quad \leq e^{L h}\|v(s-h)-X(t-h, s-h) v(s-h)\| \\
& \quad \leq e^{L h}\left\{N(t-s)+\|v\|\left(e^{N(t-s)}-1\right)+N(t-s) e^{N(t-s)}-\left[e^{N(t-s)}-1\right]\right\} .
\end{aligned}
$$

Now (19) implies (18) and then the the uniform continuity of $T^{h} v$. To prove (8) it suffices to show that

$$
\lim _{h \rightarrow 0^{+}} \sup _{t}\|X(t, t-h) v(t-h)-v(t-h)\|=0
$$


for every given $v \in C_{u}(\mathbb{R}, \mathbb{X})$. In fact, we have

$$
X(t, t-h) v(t-h)-v(t-h)=\int_{t-h}^{t} f(\xi, X(\xi, t-h) v(t-h)) d \xi .
$$

Thus from (10) we get

$$
\sup _{t}\|X(t, t-h) v(t-h)-v(t-h)\| \leq N h\left\{1+(\|v\|+N h) e^{N h}\right\} .
$$

(21) implies (20) and completes the proof of the lemma.

It is natural to consider the semigroup $\mathscr{T}$ in the context of the theory of nonlinear semigroups. It turns out that the differential operator $\mathscr{L}=$ $-d / d t+f(t, \cdot)$ constitutes the infinitesimal generator of the semigroup $\mathscr{T}$. More precisely we have the following lemma.

Lemma 2. Assume that $f$ satisfies condition $H$. Then the infinitesimal generator of the semigroup $\mathscr{T}$ associated with equation (4) is the differential operator $\mathscr{L}=-d / d t+f(t, \cdot)$ with $\mathscr{D}(\mathscr{L})=C_{u}^{1}(\mathbb{R}, \mathbb{X})$ consisting of all functions $v$ belonging together with the derivative $d v / d t$ to $C_{u}(\mathbb{R}, \mathbb{X})$.

Proof. First we show that

$$
\lim _{h \rightarrow 0^{+}} \sup _{t}\left\|\frac{1}{h} \int_{t-h}^{t} f(\xi, X(\xi, t-h) v(t-h)) d \xi-f(t, v(t))\right\|=0 .
$$

In fact, we have

$$
\begin{aligned}
\frac{1}{h} \sup _{t} & \| \int_{t-h}^{t}[f(\xi, X(\xi, t-h) v(t-h)-f(t, v(t))] d \xi \| \\
\leq & \frac{1}{h} \sup _{t} \int_{t-h}^{t} \| f(\xi, X(\xi, t-h) v(t-h)-f(\xi, v(\xi)) \| d \xi \\
& +\frac{1}{h} \sup _{t} \int_{t-h}^{t}\|f(\xi, v(\xi))-f(t, v(t))\| d \xi .
\end{aligned}
$$

From the uniform continuity of $v$ and $f$ it follows that

$$
\lim _{h \rightarrow 0^{+}} \frac{1}{h} \sup _{t} \int_{t-h}^{t}\|f(\xi, v(\xi))-f(t, v(t))\| d \xi=0 .
$$

On the other hand we have

$$
\begin{aligned}
\frac{1}{h} \sup _{t} & \| \int_{t-h}^{t}[f(\xi, X(\xi, t-h) v(t-h)-f(\xi, v(\xi))] d \xi \| \\
\leq & \frac{1}{h} \sup _{t} \int_{t-h}^{t} L\|X(\xi, t-h) v(t-h)-v(\xi)\| d \xi \\
\leq & \frac{L}{h} \sup _{t} \int_{t-h}^{t}\|X(\xi, t-h) v(t-h)-v(t-h)\| d \xi \\
& +\frac{L}{h} \sup _{t} \int_{t-h}^{t}\|v(t-h)-v(\xi)\| d \xi .
\end{aligned}
$$

Since $v$ is uniformly continuous the following equality is valid

$$
\lim _{h \rightarrow 0^{+}} \frac{L}{h} \sup _{t} \int_{t-h}^{t}\|v(t-h)-v(\xi)\| d \xi=0 .
$$


We have the estimate

$$
\begin{aligned}
\frac{L}{h} \sup _{t} & \int_{t-h}^{t}\|X(\xi, t-h) v(t-h)-v(t-h)\| d \xi \\
& \leq \frac{L}{h} \sup _{t} \int_{t-h}^{t}\left\|\int_{t-h}^{\xi} f(\eta, X(\eta, t-h) v(t-h)) d \eta\right\| d \xi \\
& \leq \frac{L}{h} \sup _{t} \int_{t-h}^{t}\left[N h\left(1+(\|v\|+N h) e^{N h}\right)\right] d \xi .
\end{aligned}
$$

From (27) it follows that (22) holds. Let us now denote by $\mathscr{A}$ the infinitesimal generator of the semigroup $\mathscr{T}$. By definition. $v \in \mathscr{D}(\mathscr{A})$ if and only if the $\operatorname{limit} \lim \left(T^{h} v-v\right) / h$ exists as $h \rightarrow 0^{+}$. In other words, $v \in \mathscr{D}(\mathscr{A})$ if and only if

$$
\lim _{h \rightarrow 0^{+}} \sup _{t}\left\|\frac{X(t, t-h) v(t-h)-v(t)}{h}-(\mathscr{A} v)(t)\right\|=0 .
$$

Let us now rewrite (28) in another form

$$
\begin{aligned}
\lim _{h \rightarrow 0^{+}} \sup _{t} \|\left\{\frac{X(t, t-h) v(t-h)-v(t-h)}{h}-f(t, v(t))\right\} \\
+\left\{f(t, v(t))+\frac{v(t-h)-v(t)}{h}-(\mathscr{A} v)(t)\right\} \|=0 .
\end{aligned}
$$

Taking into account (22) we see that (29) is equivalent to the following inequality

$$
\lim _{h \rightarrow 0^{+}} \sup _{t}\left\|f(t, v(t))+\frac{v(t-h)-v(t)}{v}-(\mathscr{A} v)(t)\right\|=0 .
$$

Since $f$ satisfies condition $H$ the function taking $t$ into $f(t, v(t))$ belongs to $C_{u}(\mathbb{R}, \mathbb{X})$. Thus $\mathscr{D}(\mathscr{A})$ consists of all functions $v \in C_{u}(\mathbb{R}, \mathbb{X})$ such that

$$
D^{-} v(t) \stackrel{\text { def }}{=} \lim _{h \rightarrow 0^{+}} \frac{v(t-h)-v(t)}{-h}
$$

belongs to $C_{u}(\mathbb{R}, \mathbb{X})$. Using the well-known results concerning such functions for the case $\operatorname{dim} \mathbb{X}=1$ we can easily prove (see e.g. [9, Chapter IX]) that in this case $D^{-} v(t)=d v(t) / d t$, i.e. $\mathscr{D}(\mathscr{A})=C_{u}^{1}(\mathbb{R}, \mathbb{X})$. From (30) it is clear that $\mathscr{A}=\mathscr{L}$. this completes the proof of the lemma.

Remark 1. Suppose that $f(t, x)=A(t) x$, where $A(t)$ is a bounded linear operator for every fixed $t$. Suppose in addition that the operator valued function $A(\cdot)$ is continuous and bounded with respect to $t$ on the whole line. Then $\mathscr{T}$ is a strongly continuous semigroup of linear operators. In this case one has no difficulty in showing that the differential operator $\mathscr{L}=-d / d t+A(t)$ is the infinitesimal generator of $\mathscr{T}$ with $\mathscr{D}(\mathscr{L}) \subseteq C_{u}^{1}(\mathbb{R}, \mathbb{X})$. From the general theory of linear semigroups [5] it follows that $\mathscr{L}^{u}$ is closed and $\mathscr{D}(\mathscr{L})$ is dense in $C_{u}(\mathbb{R}, \mathbb{X})$. If $A(\cdot)$ is uniformly continuous and bounded on $\mathbb{R}$, then from Lemma $2 \mathscr{D}(\mathscr{L})=C_{u}^{1}(\mathbb{R}, \mathbb{X})$.

In what follows we are concerned with the nonlinearly perturbed equation of

$$
\frac{d x}{d t}=f(t, x)+g(t, x)
$$


Note that if $g$ and $f$ satisfy condition $H$, then $g+f$ satisfies condition $H$ as well.

Theorem 1. Assume that $g$ and $f$ satisfy condition $H$, and there is some real $\alpha$ such that $\alpha I-\mathscr{L}$ is accretive, where $\mathscr{L}=-d / d t+f(t, \cdot)$. In addition assume that, for all sufficiently small positive $\lambda, \mathscr{R}(I-\lambda \mathscr{L})=C_{u}(\mathbb{R}, \mathbb{X})$. Then the operator $\gamma I-\mathscr{A}$ is accretive with $\gamma=\alpha+\beta$, where $\mathscr{A}=-d / d t+f(t, \cdot)+$ $g(t, \cdot), \beta$ is the Lipschitz coefficient of $g$ with respect to $x$. Furthermore, for all sufficiently small positive $\lambda, \mathscr{R}(I-\lambda \mathscr{A})=C_{u}(\mathbb{R}, \mathbb{X})$.

Proof. First we show that $(I-\lambda \mathscr{A})$ is one-to-one and onto for all sufficiently small positive $\lambda$. In fact, for every $u \in C_{u}(\mathbb{R}, \mathbb{X})$ we have to show that there is a unique $v \in C_{u}(\mathbb{R}, \mathbb{X})$ such that $(I-\lambda \mathscr{A}) v=u$ for a given sufficiently small positive $\lambda$. To this end, we write

$$
(I-\lambda \mathscr{A}) v=(I-\lambda \mathscr{L}) v-\lambda g v=u,
$$

where $(g v)(t) \stackrel{\text { def }}{=} g(t, v(t))$. It is easy to see that equation (32) is equivalent to the following one

$$
v=(I-\lambda \mathscr{L})^{-1}(u+\lambda g v)
$$

Setting

$$
F(v)=(I-\lambda \mathscr{L})^{-1}(u+\lambda g v)
$$

we see that $F$ is an operator acting on $C_{u}(\mathbb{R}, \mathbb{X})$ for a given sufficiently small positive $\lambda$. From [3, Lemma 1.2] we have

$$
\left\|(I-\lambda \mathscr{L})^{-1} u-(I-\lambda \mathscr{L})^{-1} v\right\| \leq \frac{1}{1-\lambda \alpha}\|u-v\|
$$

for all $u, v \in C_{u}(\mathbb{R}, \mathbb{X}), \lambda>0$, such that $\lambda \alpha<1$. From (35) we get the estimate

$$
\begin{aligned}
\|F(v)-F(w)\| & \leq \frac{1}{1-\lambda \alpha}\|u+\lambda g v-u-\lambda g w\| \\
& \leq \frac{\lambda \beta}{1-\lambda \alpha}\|v-w\| .
\end{aligned}
$$

Hence, for $\lambda<1 /(\alpha+\beta)$ and $\lambda \alpha<1, F$ is a contraction in $C_{u}(\mathbb{R}, \mathbb{X})$. From the Banach Fixed Point Principle it follows that $F$ has a unique fixed point which solves equation (33) and (32). Now we show that $(\gamma I-\mathscr{A})$ is accretive. In fact, we have

$$
\begin{aligned}
& \|(I+\lambda(\gamma I-\mathscr{L}-g)) u-(I+\lambda(\gamma I-\mathscr{L}-g) v \| \\
& \quad=\|[(1+\lambda \beta) I+\lambda(\alpha I-\mathscr{L})] u-[(1+\lambda \beta) I+\lambda(\alpha I-\mathscr{L}) v]+\lambda g u-\lambda g v\| \\
& \quad \geq(1+\lambda \beta)\|u-v\|-\lambda \beta\|u-v\|=\|u-v\|
\end{aligned}
$$

for all $u, v \in C_{u}(\mathbb{R}, \mathbb{X}), \lambda>0$. This completes the proof of the theorem.

Theorem 2. Suppose that all conditions of Theorem 1 are satisfied with $\alpha<0$. Then for $0 \leq \beta<-\alpha$ the perturbed equation (31) has a unique bounded solution which is globally exponentially stable.

Proof. First we show that the operator $\mathscr{L}$ defined in Theorem 1 is closed, i.e. if $u_{n} \in C_{u}(\mathbb{R}, \mathbb{X}), n=1,2, \ldots, \lim u_{n}=u$ and $\lim \mathscr{L} u_{n}=v$ as $n \rightarrow \infty$, then $v=\mathscr{L} u$. In fact, since $f$ satisfies condition $H$ we have

$$
\left\|f\left(t, u_{n}(t)\right)-f(t, u(t))\right\| \leq K\left\|u_{n}(t)-u(t)\right\|,
$$


where $K$ is the Lipschitz coefficient of $f$ with respect to $x$ from this it follows that the operator taking $u$ into $f(\cdot, u(\cdot))$ is continuous. From Remark 1 it follows that $-d / d t$ is closed. Hence the operator $\mathscr{L}=-d / d t+f(t, \cdot)$ is closed. Similarly, the operator $\mathscr{A}=-d / d t+f(t, \cdot)+g(t, \cdot)$ is closed. Let us denote by $S^{h}, h \geq 0$, the semigroup associated with equation (31), i.e.

$$
\left(S^{h} v\right)(t)=Y(t, t-h) v(t-h),
$$

where $Y(t, s) y$ is the solution to the Cauchy problem

$$
\left\{\begin{array}{l}
d y / d t=f(t, y)+g(t, y) \\
y(s)=y
\end{array}\right.
$$

Now we are in a position to apply Theorems I, II in [3] to get the following assertions

(i) $\lim \left(I-\frac{h}{n} \mathscr{A}\right)^{-n} u=S^{h} u$ as $n \rightarrow \infty$,

(ii) For all $u, v \in C_{u}(\mathbb{R}, \mathbb{X})$ the following inequality holds

$$
\left\|S^{h} u-S^{h} v\right\| \leq \exp [(\alpha+\beta) h]\|u-v\| .
$$

From Theorem 1 one sees easily that for sufficiently small positive $\lambda$ the operator $(I-\lambda \mathscr{A})^{-1}$ is a contraction. Thus it has a unique fixed point $x_{0}$. This fixed point is a bounded solution to equation (31). For every $y \in \mathbb{X}$ put $u(t)=y$ for all $t \in \mathbb{R}$. From (40) we have

$$
\left\|\left(S^{h} u\right)(h)-\left(S^{h} x_{0}\right)(h)\right\| \leq \exp [(\alpha+\beta) h] \sup _{t}\left\|u(t)-x_{0}(t)\right\| .
$$

In other words

$$
\left\|Y(h, 0) y-Y(h, 0) x_{0}(0)\right\| \leq \exp [(\alpha+\beta) h] \sup _{t}\left\|y-x_{0}(t)\right\| .
$$

Since $x_{0}(t)$ is bounded (41) shows that $x_{0}(\cdot)$ is a globally exponentially stable solution of equation (31). From the definition of $S^{h}$ it is clear that every bounded solution of equation (31) is a fixed point of $S^{h}, h>0$. Now (40) shows that such fixed points are unique and equal to $x_{0}$. This completes the proof of the theorem.

As a particular case of Theorem 2 we consider the case where $f(t, x)=$ $A(t) x$, here $A(t)$ is assumed to be a bounded linear operator for every fixed $t$. Suppose that the operator valued function $A(\cdot)$ is uniformly continuous and bounded on the whole line. In addition suppose that the evolutionary operator $X(t, s), t, s \in \mathbb{R}$, of equation (4) in this case satisfies the following condition

$$
\|X(t, s)\| \leq \exp [-\alpha(t-s)]
$$

for all $t \geq s$, where $\alpha$ is a given positive constant. A straightforward verification shows that in this case the operator $(-\alpha I-\mathscr{L})$ is accretive.

Now let us return to the linear equation (1). As shown above the class of linear equations satisfying the accretiveness mentioned in Theorems 1, 2 does not cover the class of linear equations having an exponential dichotomy or being exponentially stable. In what follows we shall make use of the spectral theory of linear semigroups to give necessary and sufficient conditions for different types of stability as well as for the existence of exponential dichotomy in terms of spectral properties of the differential operator $\mathscr{L}=-d / d t+A(t)$. 
Hereafter we assume that the operator valued function $A(\cdot)$ is continuous and bounded on $\mathbb{R}$. From Lemma 1 it follows that $\mathscr{T}$ is a strongly continuous semigroup of linear operators whose infinitesimal generator is the differential operator $\mathscr{L}$ with $\mathscr{D}(\mathscr{L})$ consisting of all $v \in C_{u}(\mathbb{R}, \mathbb{X})$ such that the following limit

$$
\lim _{h \rightarrow 0^{+}} \frac{T^{n} v-v}{h}
$$

exists.

In the case where $\operatorname{dim} \mathbb{X}<+\infty$ most of the classical results concerning the exponential dichotomy are stated in terms of the invertibility of the operator $\mathscr{L}$ (see e.g., $[4,6])$. The following theorem allows one to state them in terms of spectral properties of the operator $\mathscr{L}$.

Theorem 3. Assume that $\operatorname{dim} \mathbb{X}<\infty$ and $A(\cdot)$ is continuous and bounded on $\mathbb{R}$. Then the following assertions are true.

(i) $\lambda \in \rho(\mathscr{L})$ (with $\mathscr{D}(\mathscr{L})$ defined by (43)) if and only if the equation

$$
\frac{d x}{d t}=(A(t)-\lambda I) x
$$

has an exponential dichotomy on $\mathbb{R}$.

(ii) If $\lambda \in \rho(\mathscr{L})$, then $(\operatorname{Re} \lambda+i \zeta) \in \rho(\mathscr{L})$ for every $\zeta \in \mathbb{R}$.

Proof. If $\lambda \in \rho(\mathscr{L})$, then from the theory of linear semigroups it follows that $\mathscr{L}$ acting on $\mathscr{D}(\mathscr{L})$ defined by (43) is closed. So, we have $\mathscr{D}\left((\lambda I-\mathscr{L})^{-1}\right)=$ $C_{u}(\mathbb{R}, \mathbb{X})$ (see [9, Chapter VIII]). Modifying the proof of the classical result concerning the exponential dichotomy (see e.g. [4, Chapter IV]) one can easily prove that the above claim implies the exponential dichotomy of equation (44). Conversely, suppose that equation (44) has an exponential dichotomy with projection $P$ and positive constants $K, \alpha$. For simplicity we assume that $\lambda=0$. In this case, for every $f \in C_{u}(\mathbb{R}, \mathbb{X})$ there is a unique bounded solution $x_{f}$ to equation (44). Clearly $x_{f}$ is uniformly continuous and bounded on $\mathbb{R}$. Now we have to prove that $x_{f} \in \mathscr{D}(\mathscr{L})$ and $\mathscr{L}^{-1}$ is bounded. In fact, since $d x_{f} / d t=A(t) x_{f}-f(t)$ we have

$$
x_{f}(t)=\int_{t}^{+\infty} X(t)(I-P) X^{-1}(s) f(s) d s-\int_{-\infty}^{t} X(t) P X^{-1}(s) f(s) d s
$$

A straightforward verification shows that

$$
\frac{\left(T^{h} X_{f}\right)(t)-x_{f}(t)}{h}-f(t)=\frac{1}{h} \int_{t-h}^{t} X(t, s) f(s) d s-f(t) .
$$

To prove $x_{f} \in \mathscr{D}(\mathscr{L})$ it suffices to show that

$$
\lim \sup \left\|\frac{1}{h} \int_{t-h}^{t}[X(t, s)-I] f(s) d s\right\|=0 .
$$

In fact applying the Gronwall inequality we get the estimate

$$
\|X(a, b)-I\| \leq \exp [M]|a-b|]-1,
$$

where $M$ stands for $\sup _{t}\|A(t)\|$. From (48) we get (47), proving that $x_{f} \in$ $\mathscr{D}(\mathscr{L})$. On the other hand we have

$$
\sup _{t}\left\|x_{f}(t)\right\| \leq \int_{-\infty}^{+\infty} K \exp [-\alpha|t-s|] d s \sup _{s}\|f(s)\| \leq(2 K / \alpha)\|f\| .
$$


This completes the proof of the first assertion.

(ii) The second assertion is an immediate consequence of the first one.

Note that in the case where $\operatorname{dim} \mathbb{X}=\infty$ the invertibility of the operator $\mathscr{L}$ is not enough to guarantee the exponential dichotomy of equation (1) (see [4, 6]). However, we can find sufficient conditions for the asymptotic stability of individual solutions by using the recent results in [2]. Recall that the approximate point spectrum of $\mathscr{L}$ which we denote by $\sigma_{\mathrm{ap}}(\mathscr{L})$ consists of all $\lambda \in \mathbb{C}$ such that for every $\varepsilon>0$ there is an element $z \in \mathscr{D}(\mathscr{L}),\|z\|=1$ such that

$$
\|\lambda z-\mathscr{L} z\|<\varepsilon .
$$

Theorem 4. Assume that the operator valued function $A(\cdot)$ is continuous and bounded on $\mathbb{R}$. In addition, suppose that $\sigma_{\mathrm{ap}}(\mathscr{L})$ is disjoint from the imaginary axis. Then every solution $x(t)$ bounded on $[0,+\infty)$ is asymptotically stable, i.e. $\lim x(t)=0$ as $t \rightarrow+\infty$.

Proof. Suppose that the solution $x(t)=X(t) x$ for some $x \in \mathbb{X}$ is bounded on $[0,+\infty)$. We can choose a continuous function $\rho(t)$ such that $\rho(t)=0$ for all $|t| \geq 1$ and $\rho(0)=1$. It is clear that the function taking $t$ into $\rho(t) x(t)$ belongs to $C_{u}(\mathbb{R}, \mathbb{X})$. By definition, for $k, h \geq 0$ we have

$$
\begin{aligned}
& \left\|\left(T^{k} v\right)(t)-\left(T^{h} v\right)(t)\right\|=\|\rho(t-k) X(t) x-\rho(t-h) X(t) x\| \\
& \quad=\|[\rho(t-k)-\rho(t-h)] X(t) x\| \\
& \quad \leq|\rho(t-k)-\rho(t-h)| \sup _{t \geq-1}\|X(t) x\| .
\end{aligned}
$$

From the definition of the function $\rho(51)$ implies that

$$
\lim _{|k-h| \rightarrow 0}\left\|T^{k} v-T^{h} v\right\|=0 .
$$

We are now in a position to apply Theorem 1 in [2] to get

$$
\lim _{h \rightarrow+\infty} T^{h} v=0 .
$$

Thus

$$
\lim _{t \rightarrow+\infty}\left(T^{t} v\right)(t)=\lim _{t \rightarrow+\infty} X(t) x=0 .
$$

This completes the proof of the theorem.

Now we are going to make use of the spectral theory of linear semigroups to relate $\sigma\left(T^{h}\right)$ to $\sigma(\mathscr{L})$.

Theorem 5. Assume that the operator valued function $A(\cdot)$ is continuous and bounded on $\mathbb{R}$. Then the following assertions are true.

(i) The exponential dichotomy of equation (1) implies the hyperbolicity of $T^{h}$ for any $h \neq 0$,

(ii) The hyperbolicity of $T^{h}$ for some $h \neq 0$ implies the invertibility of the operator $\mathscr{L}$,

(iii) If $\operatorname{dim} \mathbb{X}<\infty$, the hyperbolicity of $T^{h}$ for some $h \neq 0$ is equivalent to the exponential dichotomy of equation (1) and

$$
\sigma\left(T^{h}\right)=\exp [h \sigma(\mathscr{L})]
$$


Proof. Without loss of generality we can assume that $h=1$ and equation (1) has exponential dichotomy with projection $P$ and positive constants $K, \alpha$. Then, setting

$$
\begin{aligned}
& C_{+}=\left\{V \in C_{u}(\mathbb{R}, \mathbb{X}) \mid v(t) \in \operatorname{Im}(X(t) P)(t \in \mathbb{R})\right\}, \\
& C_{-}=\left\{v \in C_{u}(\mathbb{R}, \mathbb{X}) \mid v(t) \in \operatorname{Ker}(X(t) P)(t \in \mathbb{R})\right\} .
\end{aligned}
$$

A straightforward verification shows that $C_{+}$and $C_{-}$are left invariant by $T^{1}$. In addition

$$
\begin{gathered}
C_{u}(\mathbb{R}, \mathbb{X})=C_{+} \oplus C_{-}, \\
r_{\sigma}\left(T^{1} \mid c_{+}\right)<1, \quad r_{\sigma}\left(T^{-1} \mid C_{-}\right)<1 .
\end{gathered}
$$

Thus $T^{1}$ is hyperbolic.

(ii) Applying the spectral theory of linear semigroups [5] we have

$$
\sigma\left(T^{h}\right) \supseteq \exp [h \sigma(\mathscr{L})]
$$

provided that the integral

$$
\int_{0}^{1}\left\|T^{h}\right\| d h
$$

exists. To prove (56) it suffices to show that the function taking $h$ into $\left\|T^{h}\right\|$ is continuous. In fact, by definition we have

$$
\left\|T^{h}\right\|=\sup _{\|v\| \leq 1}\left\|T^{h} v\right\|=\sup _{\|v\| \leq 1} \sup _{\xi}\|X(\xi, \xi-h) v(\xi-h)\| .
$$

From(57) a simple computation shows that

$$
\left\|T^{h}\right\|=\sup _{\xi}\|X(\xi, \xi-h)\|
$$

From (18) we can easily show that

$$
\lim _{h \rightarrow k}\left|\sup _{t}\|X(t, t-h)\|-\sup _{t}\|X(t, t-k)\|\right|=0 .
$$

This implies the continuity of the function taking $h$ into $\left\|T^{h}\right\|$. Hence (55) holds. Now if $T^{h}$ is hyperbolic then the unit circle is contained in $\rho\left(T^{h}\right)$. The invertibility of $\mathscr{L}$ follows immediately from (55).

(iii) If $\operatorname{dim} \mathbb{X}<\infty$ from Theorem 3 and assertions (i) and (ii) it follows that the invertibility of $\mathscr{L}$ is equivalent to the hyperbolicity of $T^{h}$. Applying once again Theorem 3 and taking into account (55) we get (54). The proof of the theorem is completed.

In the case $\operatorname{dim} \mathbb{X}=\infty$ from the hyperbolicity of $T^{h}$ we do not know if equation (1) has an exponential dichotomy or not. However, we can apply Theorem 4 to find individual solutions which are asymptotically stable on $[0,+\infty)$ or $(-\infty, 0]$.

Corollary 1. Assume that the operator valued function $A(\cdot)$ is continuous and bounded on $\mathbb{R}$ and $\operatorname{dim} \mathbb{X}<\infty$. Then the following assertions are true.

(i) $\sigma\left(T^{h}\right), h \neq 0$, is rotationally invariant,

(ii) Equation (1) has an exponential dichotomy if and only if the functional equation

$$
x(t)=X(t, t-h) x(t-h)+f(t),
$$


where $h \neq 0$ is a given constant, has a unique solution $x \in C_{u}(\mathbb{R}, \mathbb{X})$ for every $f \in C_{u}(\mathbb{R}, \mathbb{X})$.

Proof. (i) From Theorem 3 we get the first assertion.

(ii) From the first assertion we get the second one taking into account the fact that $1 \notin \sigma\left(T^{h}\right)$ for some $h \neq 0$ is equivalent to the exponential dichotomy of equation (1).

We now give an application of Corollary 1. Consider the functional equation

$$
x(t)=B x(t-1)+f(t),
$$

where $B$ is a nondegenerate matrix, $f \in C_{u}(\mathbb{R}, \mathbb{X})$. Suppose that $B=\exp [A]$ for some complex matrix $A$ (such a matrix always exists [4]). From Corollary 1 it follows that equation (61) has a unique solution in $C_{u}(\mathbb{R}, \mathbb{X})$ for every $f \in C_{u}(\mathbb{R}, \mathbb{X})$ if and only if equation $x^{\prime}=A x$ has an exponential dichotomy. In turn, this is equivalent to the hyperbolicity of $B$.

In the case $\operatorname{dim} \mathbb{X}=\infty$ the above claim is still true if there is an operator $A$ such that $B=\exp [A]$. Note that in this case the invertibility of $\mathscr{L}$ implies the exponential dichotomy of equation (1) (see [4, Chapter II]). Thus (54) is valid. So we can apply the above argument for this case.

Recall that $\kappa^{+}\left(\kappa^{-}\right)$is said to be the upper (lower) Bohl exponent of equation (1) defined on $J$ if it is the exact infimum (supremum) of the real numbers $\alpha$ such that

$$
\begin{aligned}
& \|X(t, s)\| \leq N \exp [\alpha(t-s)], \text { for } t \geq s,(t, s \in J), \\
& \qquad(\|X(t, s)\| \geq N \exp [\alpha(t-s)], \text { for } t \geq s,(t, s \in J),)
\end{aligned}
$$

where $N$ does not depend on $t, s$.

Proposition 1. Assume that the operator valued function $A(\cdot)$ is continuous and bounded on $\mathbb{R}$. Then one has

$$
\begin{gathered}
\kappa^{+}=\frac{1}{h} \ln r_{\sigma}\left(T^{h}\right)=\lim _{t \rightarrow+\infty}(1 / t) \ln \left\|T^{t}\right\|, \\
\kappa^{-}=-\frac{1}{h} \ln r_{\sigma}\left(T^{-h}\right)=\lim _{t \rightarrow-\infty}(1 / t) \ln \left\|T^{t}\right\|,
\end{gathered}
$$

where $h>0$.

Proof. This proposition is easily proved by using the Spectral Radius Theorem. So the details are omitted.

Theorem 6. Assume that the operator valued function $A(\cdot)$ is continuous and bounded on $\mathbb{R}$. Then the following assertions are true.

(i) Equation (1) is uniformly stable if and only if the semigroup $\mathscr{T}=\left\{T^{h}, h \geq\right.$ $0\}$ is stable, i.e.

$$
\sup _{h \geq 0}\left\|T^{h}\right\|<\infty .
$$

(ii) Equation (1) is exponentially stable if and only if the semigroup $\mathscr{T}$ is also, or equivalently,

$$
\lim _{t \rightarrow+\infty}(1 / t) \ln \left\|T^{t}\right\|<0 .
$$

Proof. (i) Applying the Banach-Steinhaus Theorem we get the first assertion. 
(ii) The second assertion is a consequence of Proposition 1.

Note that a necessary condition for equation (1) to be uniformly stable is $\kappa^{+} \leq 0$. Without any additional condition, this is not sufficient for the uniform stability. However, we can apply the generalized Hille-Yosida Theorem (see [8]) to get a necessary and sufficient condition for the uniform stability in terms of spectral properties of the differential operator $\mathscr{L}$.

Theorem 7. Assume that the operator valued function $A(\cdot)$ is continuous and bounded on $\mathbb{R}$. Then equation (1) is uniformly stable if and only if for every $n=1,2, \ldots\left(I-n^{-1} \mathscr{L}\right)^{-1}$ exists and

$$
\left\|\left(I-n^{-1} \mathscr{L}\right)^{-m}\right\| \leq C,
$$

for $n=1,2, \ldots, m=1,2, \ldots$, where $C$ is a constant not depending on $n, m$.

Proof. Since $A(t)$ is continuous and bounded on $\mathbb{R}$, the semigroup $\mathscr{T}$ is strongly continuous. Thus $\mathscr{D}(\mathscr{L})$ is dense in $C_{u}(\mathbb{R}, \mathbb{X})$. Suppose now that equation (1) is uniformly stable. From the above remark, Proposition 1, and the inclusion $(55)$ it follows that $\left(I-n^{-1} \mathscr{L}\right)^{-1}$ exists for all $n=1,2, \ldots$. From Theorem 6 and the generalized Hille-Yosida [9] we get (66). The inverse assertion is clear from the generalized Hille-Yosida Theorem. The proof of the theorem is completed.

\section{EQUATIONS ON THE HALF-LINE}

In this section we shall deal with equation (1) defined on $\mathbb{R}_{+}=[0,+\infty)$. We shall give necessary and sufficient conditions for the uniform, asymptotic uniform stability, and unstability of equation (1) by investigating spectral properties of the semigroups $\mathscr{T}=\left\{T^{h}, h \geq 0\right\}$ and $\mathscr{K}=\left\{T^{-h}, h \geq 0\right\}$ acting on suitable function spaces. Namely, we introduce the following space

$$
C_{0}=\left\{v \in C_{u}(\mathbb{R}, \mathbb{X}) \mid v(t)=0 \text { for all } t \leq 0\right\}
$$

In order to define the action of the semigroup $\mathscr{T}$ on $C_{0}$ we consider the equation

$$
\frac{d x}{d t}=B(t) x, \quad(t \in \mathbb{R}),
$$

where $B(t)=A(t)$ for $t \geq 0, B(t)=A(0)$ for $t \leq 0$.

From now on by semigroup associated with equation (1) defined on the halfline we mean the semigroup $\mathscr{T}$ associated with equation (67). Without any difficulty we can show the following results for the half-line case.

Theorem 8. Assume that the operator valued function $A(\cdot)$ is continuous and bounded on $[0,+\infty)$. Then the following assertions are true.

(i) The semigroup $\mathscr{T}$ associated with equation (1) acting on $C_{0}$ is strongly continuous.

(ii) Equation (1) is uniformly stable on $[0,+\infty)$ if and only if the semigroup $\mathscr{T}$ is stable in $C_{0}$, i.e.

$$
\sup _{h \geq 0}\left\|T^{h}\right\|_{*}<\infty,
$$

where $\|\cdot\|_{*}$ denotes the norm of operators acting on $C_{0}$. 
(iii) Denoting by $\kappa^{+}$the upper Bohl exponent of equation (1) on the half-line one has the formula

$$
\kappa^{+}=\frac{1}{h} \ln r_{\sigma}\left(T^{n}\right)=\lim (1 / t) \ln \left\|T^{t}\right\|_{*},
$$

where $h$ is a given positive constant.

Note that under the assumptions of Theorem 8 the differential operator $\mathscr{L}$ is the infinitesimal generator of the semigroup $\mathscr{T}$.

Theorem 9. Assume that the operator valued function $A(\cdot)$ is continuous and bounded on $[0,+\infty)$. Then the following assertions are valid.

(i) $\lambda \in \rho(\mathscr{L})$ if and only if the Cauchy problem

$$
\left\{\begin{array}{l}
d x / d t=(A(t)-\lambda I) x+f(t), \\
x(0)=0
\end{array}\right.
$$

has a bounded solution on $[0,+\infty)$ for every $f \in C_{0}$.

Proof. (i) Suppose that $\lambda \in \rho(\mathscr{L})$. Then the assertion is clear from the closedness of the operator $\mathscr{L}$ (see [9]). Conversely, suppose that the Cauchy problem (70) has a bounded solution on $[0,+\infty)$ for every $f \in C_{0}$. Then from the uniqueness of the solution it follows that for every $f \in C_{0}$ there is a unique solution $y \in C_{0}$ to the equation

$$
\frac{d x}{d t}=(B(t)-\lambda I) x+f(t) .
$$

To prove the existence of $(\lambda I-\mathscr{L})^{-1}$ we have to show that $y \in \mathscr{D}(\lambda I-\mathscr{L})$. Without loss of generality we assume that $\lambda=0$. Thus we have to show

$$
\lim _{h \rightarrow 0^{+}}\left[\frac{T^{h} y-y}{h}-f\right]=0
$$

which we write in another form

$$
\lim _{h \rightarrow 0^{+}} \sup _{t}\left\|\frac{1}{h} \int_{t-h}^{t} X(t, s) f(s) d s-f(t)\right\|=0 .
$$

To prove (73) it suffices to show that

$$
\lim _{h \rightarrow 0^{+}} \sup _{t-h \leq \xi \leq h}\|X(t, \xi)-I\|=0 .
$$

In turn (74) follows immediately from (18). Now we have to show that $\mathscr{L}^{-1}$ is bounded. To this purpose, we need a minor modification of Theorem 5.2 [4, Chapter III] which says that in this case $\kappa^{+}<0$. This implies the boundedness of $\mathscr{L}^{-1}$, completing the proof of the first assertion.

(ii) The second assertion follows immediately from the first one and the above-mentioned fact that if $\lambda \in \rho(\mathscr{L})$ then $\kappa^{+}<\operatorname{Re} \lambda$.

Theorem 10. Assume that the operator valued function $A(\cdot)$ is bounded and continuous on $[0,+\infty)$. Then the spectral mapping theorem is valid for the semigroup associated with equation (1), i.e.

$$
\sigma\left(T^{h}\right)=\exp [h \sigma(\mathscr{L})], \quad(h>0) .
$$


Proof. A simple computation shows that

$$
\left\|T^{h}\right\|_{*}=\sup _{t \geq h}\|X(t, t-h)\| .
$$

From (76) we have no difficulty in showing that the function taking $h$ into $\left\|T^{h}\right\|_{*}$ is continuous. Thus we are in a position to apply the spectral theory of linear semigroups [5], to get the inclusion

$$
\sigma\left(T^{h}\right) \supseteq \exp [h \sigma(\mathscr{L})], \quad(h>0) .
$$

We now prove the inverse inclusion. In fact, clearly, $0 \in \sigma\left(T^{h}\right)$. In addition, from Theorem 9 it follows that

$$
\sigma(\mathscr{L})=\left\{z \in \mathbb{C} \mid \operatorname{Re} z \leq \kappa^{+}\right\} .
$$

From (78), (79), and Theorem 8 we get the assertion. The proof is completed.

Corollary 2. Assume that the operator valued function $A(\cdot)$ is continuous and bounded on $[0,+\infty)$. Then for every given positive number $h$ the following assertions are true.

(i) $\sigma\left(T^{h}\right)$ is the closed disk $\left\{z \in \mathbb{C}|| z \mid \leq \exp \left[h \kappa^{+}\right]\right\}$. In particular, $\sigma\left(T^{h}\right)$ is rotationally invariant.

(ii) Equation (1) is asymptotically uniformly stable if and only if the following functional equation

$$
\left\{\begin{array}{l}
x(t)=X(t, t-h)+f(t), \quad(t \geq 0) \\
x(\xi)=0,-h \leq \xi<0
\end{array}\right.
$$

has a unique solution which is bounded and uniformly continuous on $[0,+\infty)$ for every $f \in C_{0}$.

Proof. The corollary is an immediate consequence of Theorem 10.

Note that in the case $\sigma\left(T^{h}\right)$ contains the unit circle, or equivalently, $\sigma(\mathscr{L}) \supseteq$ $i \mathbb{R}$. From Corollary 2 we cannot deduce any claim concerning the exponential stability of equation (1). If equation (1) has a nontrivial exponential dichotomy on the half line it is not hard to show that $\sigma(\mathscr{L}) \supseteq i \mathbb{R}$ but $\sigma_{\text {ap }}(\mathscr{L}) \cap i \mathbb{R}=\varnothing$. For this reason we state here the half-line version of Theorem 4 which is not a trivial sufficient condition for the asymptotic stability of individual solutions.

Theorem 11. Assume that $A(\cdot)$ is bounded and continuous on the half-line. In addition, suppose that $\sigma_{\mathrm{ap}}(\mathscr{L})$ is disjoint from the imaginary axis. Then every bounded solution of equation (1) is asymptotically stable.

Proof. The proof is carried out in the same way as in Theorem 4.

We now derive the half-line version of Theorem 7 which can be similarly proved.

Theorem 12. Assume that the operator valued function $A(\cdot)$ is continuous and bounded on the half-line. Then equation (1) is uniformly stable if and only if $\left(I-n^{-1} \mathscr{L}\right)^{-1}$ exists for every $n=1,2, \ldots$ and there is a positive constant $C$ not depending on $n, m$ such that

$$
\left\|\left(I-n^{-1} \mathscr{L}\right)^{-m}\right\|_{*} \leq C
$$

for all $n=1,2, \ldots, m=1,2, \ldots$. 
Now let us consider the unstability of equation (1). To do this we introduce a new space $C_{u}\left(\mathbb{R}_{+}, \mathbb{X}\right)$ consisting of all $\mathbb{X}$-valued bounded and uniformly continuous function on $\mathbb{R}_{+}=[0,+\infty)$. It is clear that $C_{u}\left(\mathbb{R}_{+}, \mathbb{X}\right)$ is a complex Banach space. Consider the semigroup $\mathscr{K}=\left\{T^{-h}, h \geq 0\right\}$ acting on $C_{u}\left(\mathbb{R}_{+}, \mathbb{X}\right)$ as follows

$$
\left(T^{-h} v\right)(t)=X(t, t+h) v(t+h), \quad(t \geq 0),
$$

for all $v \in C_{u}\left(\mathbb{R}_{+}, \mathbb{X}\right)$.

Lemma 3. Assume that $A(\cdot)$ is bounded and continuous on the half-line. Then the semigroup $\mathscr{K}$ is strongly continuous. In addition, its infinitesimal generator is the differential operator $-\mathscr{L}=d / d t-A(t)$.

Proof. The lemma is proved in the same way as Lemma 1.

Proposition 2. Assume that $A(\cdot)$ is continuous and bounded on the half-line. Then, denoting by $\kappa^{-}$the lower Bohl exponent of equation (1) on the half-line, one has

$$
\kappa^{-}=-\frac{1}{h} \ln \left\|T^{-h}\right\|_{\#,}, \quad(h>0) .
$$

where $\left\|T^{-h}\right\|_{\#}$ denotes the norm of the operator $T^{-h}$ acting on $C_{u}\left(\mathbb{R}_{+}, \mathbb{X}\right)$.

Proof. The proposition follows immediately from the definition of the lower Bohl exponent and the Spectral Radius Theorem.

Theorem 13. Assume that $A(\cdot)$ is continuous and bounded on the half-line. Then the following assertions are valid.

(i) $\lambda \in \rho(-\mathscr{L})$ if and only if for every $f$ continuous and bounded on $\mathbb{R}_{+}$the equation

$$
\frac{d x}{d t}=(A(t)+\lambda I) x+f(t)
$$

has a unique bounded solution on $\mathbb{R}_{+}$.

(ii) If $\lambda \in \rho(-\mathscr{L})$, then $\eta \in \rho(-\mathscr{L})$ for every $\eta$ satisfying $\operatorname{Re} \eta \geq \operatorname{Re} \lambda$.

Proof. (i) Suppose that $\lambda \in \rho(-\mathscr{L})$. Since $\mathscr{L}$ is closed we get $\mathscr{D}\left((\lambda I+\mathscr{L})^{-1}\right)$ $=C_{u}\left(\mathbb{R}_{+}, X\right)$. A minor modification of Theorem 3.3, in [4, Chapter IV] shows that equation (83) has an exponential dichotomy with trivial projection $P=0$. Thus for every $f$ continuous and bounded on $\mathbb{R}_{+}$equation (83) has a unique bounded solution on $\mathbb{R}_{+}$. Conversely, suppose that equation (83) has a unique bounded solution on $\mathbb{R}_{+}$for every $f$ bounded and continuous on $\mathbb{R}_{+}$. We have to show that $(\lambda I+\mathscr{L})^{-1}$ exists and bounded. Without loss of generality we assume that $\lambda=0$. From our assumptions it follows that equation (83) has an exponential dichotomy with trivial projection. Thus the unique bounded solution $x_{f}$ of equation (83) has the form

$$
x_{f}(t)=-\int_{t}^{\infty} X(t, s) f(s) d s .
$$

It is clear that $x_{f} \in C_{u}\left(\mathbb{R}_{+}, \mathbb{X}\right)$. To prove the existence of $\mathscr{L}^{-1}$ as a mapping from $C_{u}\left(\mathbb{R}_{+}, \mathbb{X}\right)$ to $\mathscr{D}(\mathscr{L})$ we have to show that for every $f \in C_{u}\left(\mathbb{R}_{+}, \mathbb{X}\right)$

$$
\lim _{h \rightarrow 0^{+}}\left[\frac{T^{-h} x_{f}-x_{f}}{h}-f\right]=0 \text {. }
$$


We write (85) in another form

$$
\lim _{h \rightarrow 0^{+}} \sup _{t}\left\|\frac{1}{h} \int_{t}^{t+h} X(t, s) f(s) d s-f(t)\right\|=0 .
$$

From the uniform continuity of $f$ it suffices to show that

$$
\lim _{h \rightarrow 0^{+}} \sup _{t}\left\|\frac{1}{h} \int_{t}^{t+h}[X(t, s)-I] f(s) d s\right\|=0 .
$$

In turn, (87) follows immediately from a result similar to (18). Thus $\mathscr{L}^{-1}$ takes $f$ into $x_{f} \in \mathscr{D}(\mathscr{L})$. The boundedness of $\mathscr{L}^{-1}$ follows from (84).

(ii) The second assertion follows from the exponential dichotomy with trivial projection of equation (83).

Theorem 13. Assume that $A(\cdot)$ is continuous and bounded on the half-line. Then the spectral mapping theorem is valid for the semigroup $\mathscr{K}$, i.e.

$$
\sigma\left(T^{-h}\right)=\exp [h \sigma(-\mathscr{L})] .
$$

Proof. First note that $0 \in \sigma\left(T^{-h}\right)$. Similarly to Theorem 10, applying the spectral theory of linear semigroups we get

$$
\sigma\left(T^{-h}\right) \supseteq \exp [h \sigma(-\mathscr{L})] .
$$

Applying Theorem 12 we can show that the inverse inclusion is also true. The proof of the theorem is completed.

Corollary 3. Assume that the operator valued function $A(\cdot)$ is continuous and bounded on the half-line. Then for every given positive constant $h$ the following assertions are true:

(i) $\sigma\left(T^{-h}\right)$ is rotationally invariant.

(ii) The lower Bohl exponent of equation (1) is positive if and only if $1 \notin$ $\sigma\left(T^{-h}\right)$, or equivalently, the following functional equation

$$
x(t)=X(t, t+h) x(t+h)+f(t), \quad(t \geq 0)
$$

is uniquely solvable in $C_{u}\left(\mathbb{R}_{+}, \mathbb{X}\right)$ for every $f \in C_{u}\left(\mathbb{R}_{+}, \mathbb{X}\right)$.

\section{ACKNOWLEDGMENT}

The author would like to thank the referee for valuable suggestions and P. P. Zabreiko for useful discussions.

\section{REFERENCES}

1. B. Aulbach, Nguyen Van Minh, and P. P. Zabreiko, A generalization of monodromy operator and its applications, preprint.

2. C. K. J. Batty and Vu Quoc Phong, Stability of individual elements under one-parameter semigroups, Trans. Amer. Math. Soc. 322 (1990), 805-818.

3. M. G. Crandall and T. M. Liggett, Generation of semigroups of nonlinear transformations on general Banach spaces, Amer. J. Math. 93 (1971), 265-298.

4. Ju. L. Daleckii and M. G. Krein, Stability of solutions of differential equations in Banach spaces, Amer. Math. Soc., Providence, R.I., 1974.

5. E. Hille and R. Phillips, Functional analysis and semigroups, Amer. Math. Soc. Colloq. Publ., vol. 31, Amer. Math. Soc., Providence, RI, 1957. 
6. J. L. Massera and J. J. Schaffer, Linear differential equations and function spaces, Academic Press, New York and London, 1966.

7. Nguyen Van Minh, Semigroups and linear nonautonomous differential equations on the half line, Dokl. Akad. Sci. Belarus 37 (1992), no. 1, 19-22. (Russian)

8. __ Nonlinear semigroups and nonlinear nonautonomous differential equations, Dokl. Akad. Sci. Belarus 37 (1993), no. 2, 113-117. (Russian)

9. K. Yosida, Functional analysis, Springer-Verlag, Berlin-Göttingen-Heidelberg, 1965.

10. P. P. Zabreiko and Nguyen Van Minh, Group of characteristic operators and its applications in the theory of linear differential equations, Dokl. Russian Acad. Sci. 324 (1992), no. 1, 24-28. (Russian)

Department of Mathematics and Mechanics, Byelorussian State University, Minsk 220 080, BELARUS

Current address: Steklov Institute of Mathematics, Vavilov Str. 42, Moscow, Russia 